\title{
The Research on WLAN Technology of Chain Network Based on Monitoring Information Transmission of Catenary
}

\author{
Wu Chunmeia , Li Guilin, Wang Shaowei and Lan Xueyan \\ College of electrical information, Dalian Jiaotong University, Dalian 116028, Liaoning Province, China
}

\begin{abstract}
The structure of railway catenary line is "long chain". Considering each catenary as a wireless node, it will form the chain wireless network that solves the transmission problem of monitoring data on railway catenary line. Using token method to manage the distribution of channel resources can optimize the structure of "long chain". The local system decomposes several subnet class units which consist of one or more nodes. Then the token is transmitted in denomination of subnet. The system structure is composed of multiple subnets connected in series, making the route more flexible. The network can self-repair and prevent system breakdown due to node failures. The stability of system will be enhanced. The monitoring data can be sent in parallel, and the network efficiency becomes higher. Using token as control information, nodes will not interfere with each other, thus achieving orderly utilization of the channel.
\end{abstract}

\section{Introduction}

The catenary line is composed of a group of poles and two ends of power supply workshops, electric power communication covers only the power supply workshops, but the whole transmission line corridor is the blank of coverage, as shown in figure 1 . In order to transmit the monitoring information to the power supply workshops, it is necessary to construct the communication channel of transmission line corridor.

Because of long distance and high-voltage of the catenary line, it is very difficult to set up the monitoring communication network by laying cables, and the economy could not afford, so wireless communication is usually adopted ${ }^{[1]}$. The wireless communication technology for on-line monitoring device of transmission line includes WiFi, WiMax, wireless Mesh network technology and wireless pubic network. WiFi and ZigBee are not suitable for the long chain structure of transmission lines. At present, the public network transmission is feasible, however, due to the limited coverage of the public network and the high running cost of the system, it is difficult to meet the requirements of the power system too $^{[2]}$.

Chain transmission network based on the wireless Mesh has the characteristics of dynamic self-organization, self-repairing and self-balance ${ }^{[3]}$. On-line monitoring access points of catenary line require a wide coverage, and communication equipment should operate in harsh environments. However, the communication bandwidth and real-time of data transmission requirements are not high. This makes wireless Mesh technology become the mainstream technology of transmission line on-line monitoring communication. Using the advantages of wireless Mesh network technology, transmit monitoring data to power supply workshops, then transmit the data to monitoring center through the optical communication system. This method avoids the complexity of communication line wiring, and could also be installed in harsh environments ${ }^{[4]}$.Wireless chain network is suitable for transmission line communication system, the network mode and points access are flexible. This provides a data transmission channel for power network monitoring. The transmission efficiency is improved, the network security is strengthened, and the reliability of the power communication network is ensured. It is a great significance to the safe operation of railway power supply ${ }^{[5-6]}$.

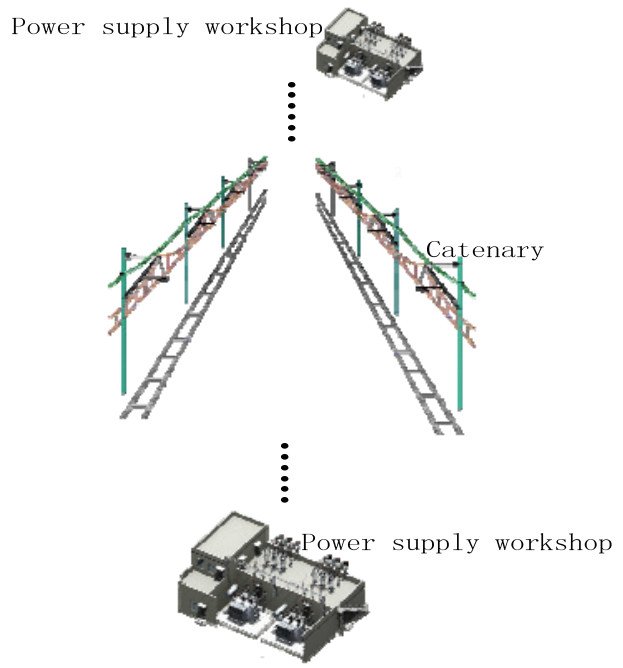

Figure 1. Catenary line's diagram of distribution

aCorresponding author: m18524406315@163.com 


\section{Physical structure of chain wireless network}

The distance between the poles of the catenary line is about 50 meters, and it is linear distribution, which provide a natural condition for short distance wireless multi hop transmission. This paper uses the scheme of catenary line monitoring communication based on wireless Mesh: The monitoring devices collect the relevant state information of catenary line and transfer to the wireless Mesh routing node, then the Mesh nodes transmit the information to the sink (power supply workshop) through wireless multi hop. Finally, upload the information to the monitoring center.

The backbone line data transmission module uses 802.11 a standard of $5.8 \mathrm{GHz}$, which has 12 non overlapping channels to facilitate the deployment of large-scale networks. Monitoring device information access module is configured as $802.11 \mathrm{~b} / \mathrm{g}$ standard of $2.4 \mathrm{GHz}^{[7]}$. Physical structure of the chain wireless network is shown in figure 2 .

In practice, we can adjust the transmitting power of wireless module to control the coverage of nodes and reduce the interference of the neighbor nodes. In chain wireless network, we control the transmitting power of nodes and make the coverage is two hop nodes, which means that the nodes can only listen to the idle condition of the channel within two hops and the nodes outside the two hop range can transmit information without producing interference simultaneously.

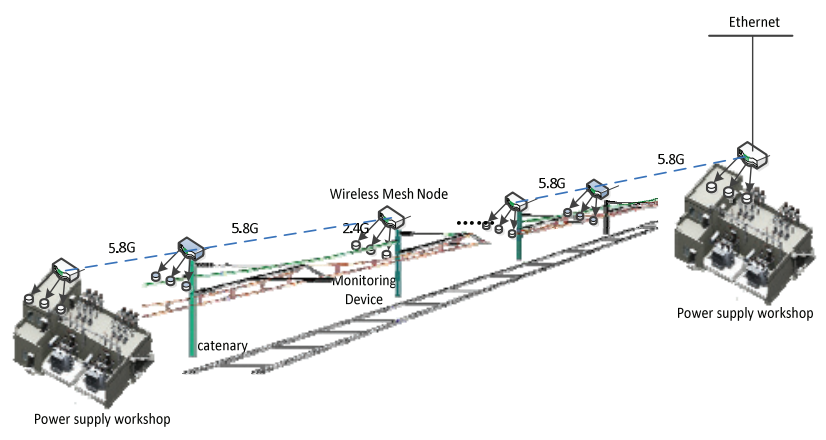

Figure 2. Physical structure of chain wireless network

\section{The topological structure of chain wireless network based on token}

In this paper, a special topological structure of multi subnet in series for "chain structure" is presented, which belongs to the type of Mesh and is composed of a plurality of wireless communication networks that are connected in series, each sub network including one or more peer wireless monitoring nodes ${ }^{[8]}$.

The chain wireless network consists of the initial power supply workshop, the main node of the token-ring which has data to transmit, the next main node, the common nodes and terminal power supply workshop. A main node of token-ring and two common nodes forms a wireless communication subnet. A plenty of subnets in series connection form a long chain. The initial power supply workshop completes the procedure of initialization, so that each node knows its predecessor and successor nodes; the main node of token-ring is the node which has monitoring data to transmit. Before transmission, it listens to the idle condition of channel within two hop range, and declares to be the main node of token-ring, then it makes up a token ring to transmit the monitoring data; The next main node is the main node of the next sub token-ring network, which is similar to the main node of the token-ring which has data to transmit; The common node plays a role of relay in the information transmission, it is the intermediate node in the transmission process; The terminal power supply workshop is the node at the end of catenary line, and the data information on the line will be collected to the power supply workshop and be handled finally.

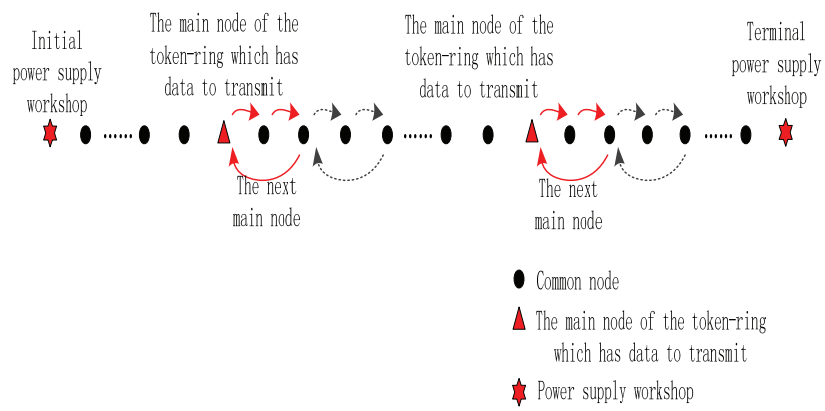

Figure 3. The topological structure of chain wireless network based on token

\section{Working mode of token in wireless network}

\subsection{Initialization, make nodes know their predecessor and successor nodes}

Power supply workshop monitoring center nodes (the two ending nodes of the long chain) are responsible for the generation, taking back and management of tokens. Each node is assigned a unique address of the whole network as its own identity, and has an initial neighbor table which is used to determine the destination address of the next hop. The center node receives the remote command to start and generate a "Neighbornode-detection" token. The token carries the address information of the current node. In the form of single hop, the token is transmitted along the nodes of initial path. The nodes read the value of SA when they receive the token ${ }^{[9]}$, and record it as their predecessor node. When the token arrives at the terminal of the line, nodes transmit the detection token back according to the original path and the token will be token back by center node ${ }^{[10]}$.Complete the initialization, and form a neighbor node table for future node operation.

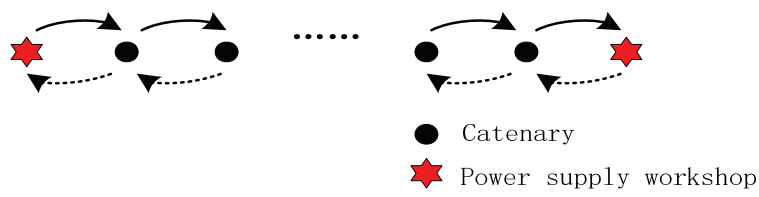

Figure 4. Initialization process of nodes access to electricity

4.2 When node has data to transmit, the procedure of creating a logical ring 
After initialization, the node enters the FLT state. If the node needs to transmit data and the channel of its adjacent two hop range is idle, then the node enters the SFR state and keeps on listening, if the channel is still idle, it declares to be the main node of the token-ring and sends a solicit_successor token to invite its next hop node to join the token-ring. Then the node enters SEK state, waiting for the response of other nodes. When the next hop node receives the solicit_successor token sent by the node, then it enters the SRP state for random back-off. If it receipts the information of the existence of other token ring during the back-off time, then it returns to the FLT state and waits for the next opportunity to join; If there is no response of other nodes, then it replies the set successor token to respond and receives the token with right to send data which is sent by the invitation node. The one hop successor node which join the token ring successfully has the right to invite the two hop successor node of the main node to join the token ring. With the token passing periodically, it makes the nodes of the two hop range of main node join the token ring orderly.

When the data frame is transmitted to the two hop successor node of the main node, the two hop successor node sends common token to the main node, then the main node reads the value of GenSeq of the frame. When the value of GenSeq is 1 (the token is transmitted one circle on the ring), the main node will take back the token and send the set successor token to the successor node of its two hop. After its two hop successor node receives the confirmation signal and the channel of its two hop range is idle, it declares to be the new main node of the token ring. In accordance with the above steps, a subnet is established. A plurality of subnets which are connected in series realize the transmission of data to the power supply workshop.

Because the data transmission is random, there may be multiple tokens on the whole line, that is, there may be multiple subnets on the whole line to transmit data at the same time. However, the channel of the main node's adjacent two hop range is idle as the logical ring is set up, so there is no interference during each subnet transmitting the data. This not only ensures the correctness of data transmission, but also reduces the delay of data transmission.

\subsection{The process of nodes joining the token-ring}

The process of nodes joining the token-ring is based on the four steps handshake mechanism.

After a node holds the token, it sends a solicit_successor token and asks for the nodes whose address is between the current node and the next node in the logical order to join the token-ring.

Node A broadcasts the solicit_successor token that contains the address information of Node C. In order to avoid the collision of the plurality of application nodes' joining responses, after Node B hears the solicit_successor, it will choose a random back-off time. If Node $\mathrm{B}$ hears the set_successor token that is sent by other nodes during the back-off time, it will give up this application. Otherwise, when the back-off time is over, the joining application will be issued immediately, and Node $\mathrm{C}$ will be recorded as its probable successor node. After Node A receives the set_successor token sent by Node B, it will record Node B as its probable successor node and send the set predecessor token. After Node B receives the set predecessor token, it will record Node A as its probable predecessor node and send the set predecessor token to Node $\mathrm{C}$ in order to close the token ring.

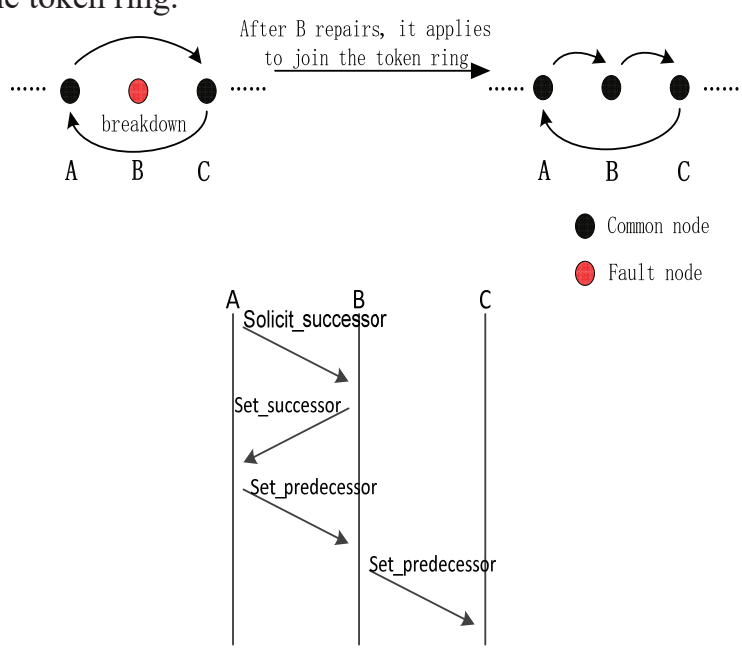

Figure 5. Nodes join token ring

\subsection{The process of nodes leaving the token-ring}

The protocol needs to support the recovery mechanism of token ring network, because the nodes on the token ring may break down. The process of node leaving the network is shown in figure 6. If Node B needs to apply to leave the token ring, it will wait for the token being held and notify its predecessor node spontaneously. Specific steps are as follows: Node B sends the set successor token to its predecessor node (Node A), which contains the MAC address of its successor node (Node C), and declares it will leave the token ring. Node A sends the set_predecessor token to Node C, enters the listening state and starts the token transfer timer. If the timer does not overflow and Node A hears the implied confirmation signal, it is considered that the token ring is closed successfully. And nodes will upload their local connection table.

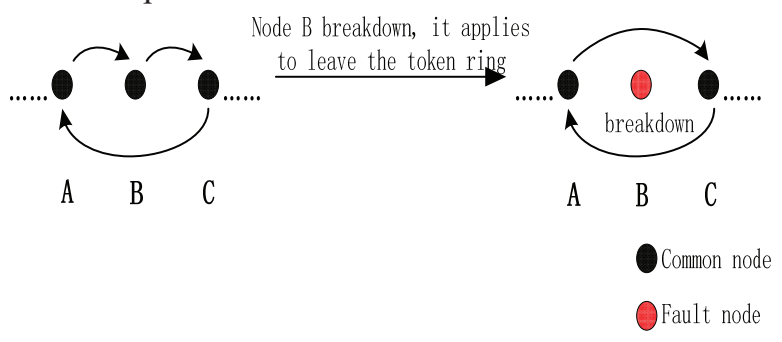

Figure 6. Nodes leave token ring

\section{The maintenance of token ring network}

\subsection{Fault handling}

(1) One hop successor node of the main node breaks down If the main node of token ring (Node C) does not hear valid message, it will send the token again to the same successor node (Node D). After two successive failures, 
Node C considers Node D is broken-down and then sends a solicit_successor packet in order to find the address of Node E which is the successor node of the broken-down Node D. If Node $\mathrm{C}$ receives the set_successor response, it shows that the next node of Node D (Node E) can work normally, so Node $\mathrm{C}$ skips the broken-down point Node D, and connects with the new successor Node E. If Node C doesn't receive the set_successor response, it can retry. If it fails again, it indicates that the network node is brokendown or the receiver of the node broken-down. Node $\mathrm{C}$ will stop working right now, and listen to the condition of the bus.

If the main node of token ring (Node C) connects with its two hop successor node (Node E), Node C will take the token back after one circle of token passing, and send a confirmation signal to Node E. After Node E receive the confirmation signal, if it hears the channel of its adjacent two hop range is idle, then it declares to be the new main node of token ring and forms a new token ring with Node F and Node G.

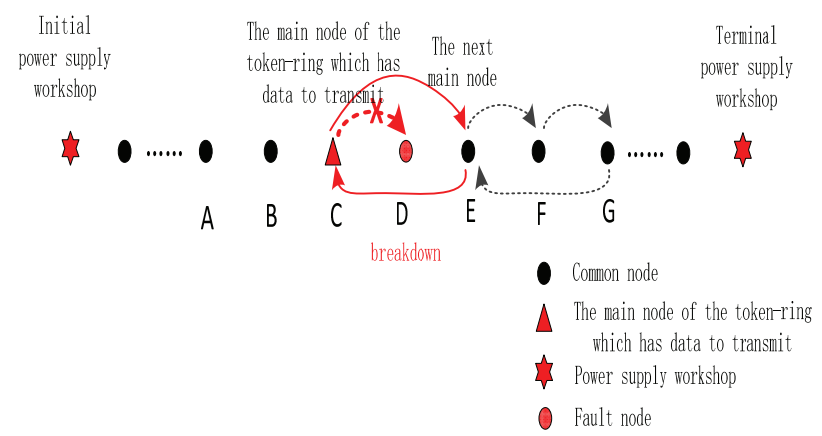

Figure 7. Main node's one hop successor node breaks down

(2) Two hop successor node of the main node breaks down

If one hop successor node (Node D) of the main node (Node C) works normally, and Node C can hear a valid message, then Node $\mathrm{C}$ convers to the state of listening. And the token is passed by Node D, if Node D which sends the token doesn't hear the valid message, it will send the token to the same one hop successor node (Node E) again. After two successive failures, Node D considers Node E is broken-down and send a set predecessor packet which contains its own address. Node C and Node D form a new token ring which contains two nodes.

If the main node of token ring (Node C) and its one hop successor node (Node D) form a token ring which has two nodes successfully, Node $\mathrm{C}$ will take the token back after one circle of token passing and send a confirmation signal to Node D. After Node D receives the confirmation signal, if it hears the channel of its adjacent two hop range is idle, then it declares to be the new main node of token ring, skips the broken-down point Node $\mathrm{E}$ and forms a new token ring with

node

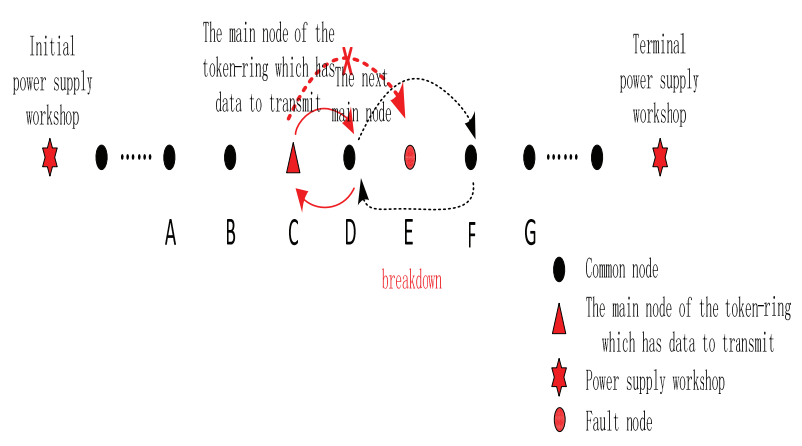

Figure 8. Main node's two hop successor node breaks down

\subsection{The elimination of multiple tokens}

The chain wireless transmission protocol should be able to detect the situation of multiple tokens existing in each subnet, and could take some measures to eliminate multiple tokens. Use the priority of the token to eliminate the low priority token, so that only one token is passed in a token ring. The more nodes, the higher priority of the token ring. If the number of nodes of the ring is the same, the older age of token ring, the higher priority. In the case of token loss, the age of the regeneration token will be added 2 based on the age of local ring. So the priority of new token will be higher than original token, thus the old token will be eliminated.

\section{Performance analysis of the chain wireless network}

Literature $^{[11]}$ analyze the data flow of digital substation communication network and simulate the network performance. Literature ${ }^{[12]}$ analyzes and simulates the performance of the wireless token ring protocol and proposes the optimization algorithm. According to the transmission process and characteristics of the power supply workshop communication network monitoring data flow, in this paper, the traditional token ring protocol is improved. The system is decomposed into several subnets which connect in series, based on that, we mainly analyze the network performance indexes such as the information transmission delay, the information throughput and the number of nodes that can be accommodated by the system.

In the electric power monitoring system, the length of the monitoring data depends on the negative exponential distribution. Usually the monitoring data is loaded in the message segment of frame whose length is less than or equal to $b_{0}$, in this case, it can be transferred effectively.

Assume that the length of the message segment is a random variable $X$, and its density function is $\mathrm{f}(x)=$ $\mu e^{-\mu x}$. The length of frame follows a negative exponential distribution and a fixed length of service time, it is a random variable $\mathrm{L}$, and its density function is $\mathrm{f}(l)$. That is, some frames in the range of $\mathrm{h} \sim \mathrm{h}+b_{0}$ depends on the negative exponential distribution and their density functions are $f_{1}(l)$, while the other frames whose length are fixed values $\mathrm{h}+b_{0}$, their density functions are $f_{2}(l)$.

where 


$$
\begin{gathered}
f_{1}(l)=\left\{\begin{array}{cl}
\frac{\mu e^{-\mu(l-h)}}{1-e^{-\mu b_{0}}}, & h<l<h+b_{0} \\
0, & l<h \text { orl }>h+b_{0}
\end{array}\right. \\
f_{2}(l)=\delta\left(l-\left(h+b_{0}\right)\right)
\end{gathered}
$$

The length of frame $\mathrm{L}$ to loop bandwidth $\mathrm{C}$ ratio is the frame transmission time $\mathrm{S}$. The expressions are obtained by calculation as follow:

$$
\begin{gathered}
\mathrm{E}(S)=\frac{1+\mu h-e^{-\mu b_{0}}}{\mu c} \\
\mathrm{E}\left(S^{2}\right)=\frac{1+(1+\mu h)^{2}-2\left(1+\mu b_{0}+\mu h\right) e^{-\mu b_{0}}}{(\mu c)^{2}}
\end{gathered}
$$

The wireless chain transmission network is composed of $m$ subnets which connect in series, and each subnet consists of three nodes. The token is passed on the token ring nodes one by one in one way in each power supply workshop. After the information frame arrives at the node, it participates in the queue and waits for the token. The node which acquires the token can send only one information frame and follow the principle of first come first served. Assume that the information frame which arrives at node i depends on Poisson distribution, and the average arrival rate is $\lambda_{i}(i=0,1,2)$. Then token ring is $\mathrm{M} / \mathrm{G} / 1$ queue system. Assume that each subnet has the same performance, so we first analyze the performance of a single subnet.

Average waiting time of information frame in subnet:

$$
\mathrm{E}(D)=\frac{\lambda E\left(S^{2}\right)}{2(1-\rho)}
$$

Where $\lambda=\lambda_{0}+\lambda_{1}+\lambda_{2}, \quad \rho=\lambda \mathrm{E}(S)$ is loop busy probability.

The transmission delay of the information frame of the subnet is the time interval form the information frame reaching a node to being sent out, its mathematical expectation is:

$$
\mathrm{E}(W)=E(D)+E(S)
$$

The time of one circle of token passing on the ring:

$$
B_{r}=\sum_{i=0}^{2}\left[\rho_{i} S+\left(1-\rho_{i}\right) l_{t}\right]=\rho S+(1-\rho) l_{t}(8)
$$

Where $\rho_{i}=\lambda_{i} E(S)$ is the busy probability of node i, $l_{t}$ is the passing time of idle token.

Define the number of information bits transmitting on the subnet in unit time as the information throughput of subnet.

$$
\mathrm{H}=\frac{\frac{\left(1-e^{\left.-\mu b_{0}\right) \rho_{i}}\right.}{\mu}}{\rho E(S)+(1-\rho) l_{t}}
$$

According to IBM TOKEN RING frame format, the length of monitoring segment is 336bit and idle token is 18bit. Assume that average length of user information is $\frac{1}{\mu}=1376$ bit and loop bandwidth is $25 \mathrm{Mb} / \mathrm{s}$.

According to the above formula, simulate with matlab, the performance analysis curves of the protocol of chain wireless network which consists of multi subnets in series are as follow.

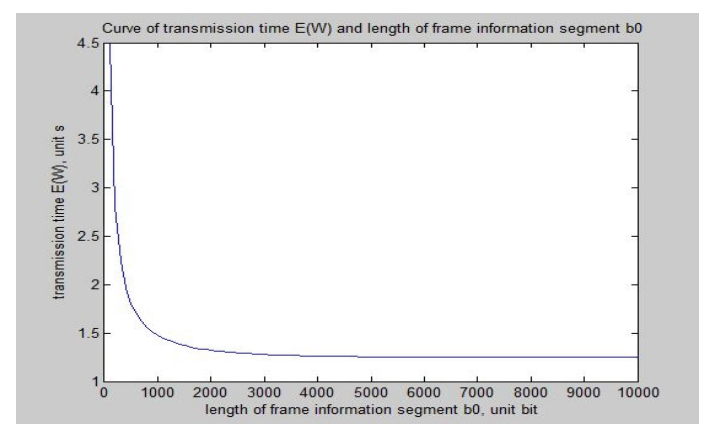

Figure 9. Curve of transmission time $\mathrm{E}(\mathrm{W})$ and length of frame information segment b0

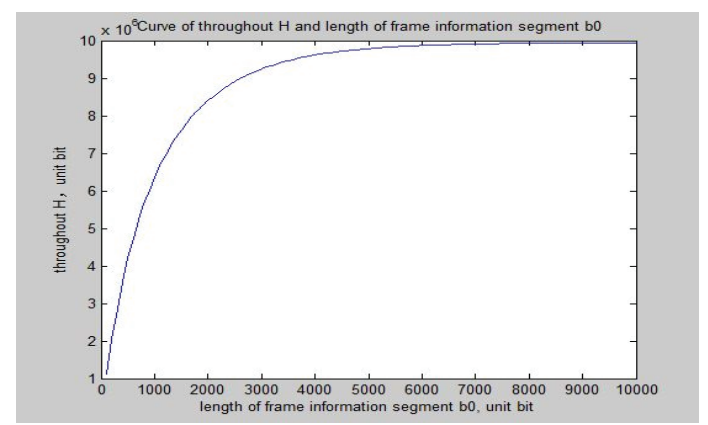

Figure 10. Curve of throughout $\mathrm{H}$ and length of frame information segment b0

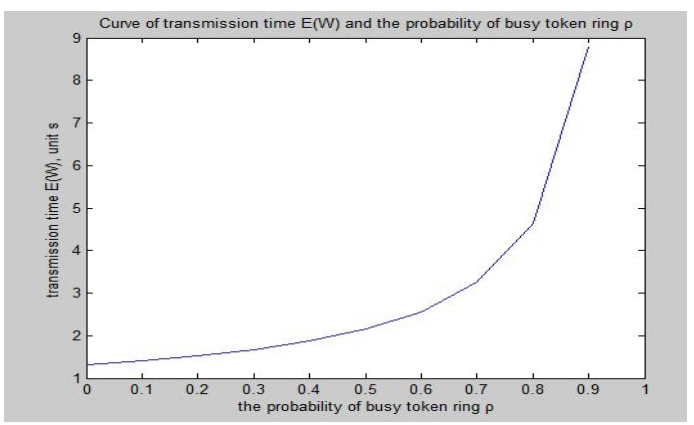

Figure 11. Curve of transmission time $\mathrm{E}(\mathrm{W})$ and the probability of busy token ring $\rho$

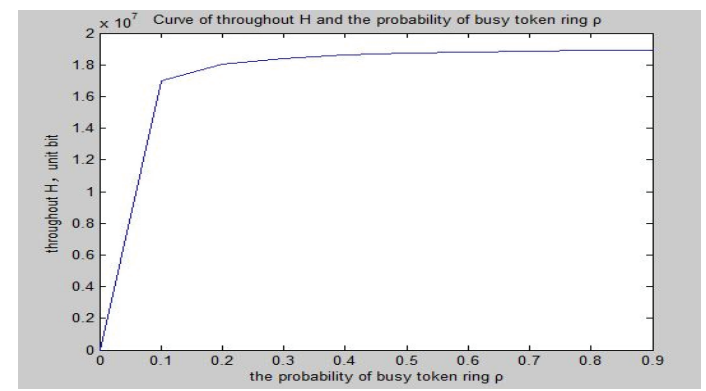

Figure 12. Curve of throughout $\mathrm{H}$ and the probability of busy token ring $\rho$

As it is shown in Figure 9 and Figure 10, for the maximum information throughout $\mathrm{H}$ and the minimun transmission delay $\mathrm{E}(\mathrm{W})$, the optional maximum length of message segment is different, and the latter is often less than the former. When $b_{0}>2000 \mathrm{bit}$, the growth rate of information throughout has a sharp decline, while the transmission delay $\mathrm{E}(\mathrm{W})$ no longer reduces. $b_{0}$ should take account of these two parameters, in order to obtain the smaller transmission delay and the larger information throughout. Therefore, the length of information segment of the frame $b_{0}$ should not be greater than 200 bit. 
As it is shown in Figure 11 and Figure 12, when the loop busy probability $\rho$ is bigger than 0.8 , the transmission delay $\mathrm{E}(\mathrm{W})$ grows rapidly. Compromise the transmission delay $\mathrm{E}(\mathrm{W})$ and the information throughout $\mathrm{H}$, the ideal value of $\rho$ is in the range of $0.5 \sim 0.8$. The relation of the number of nodes $\mathrm{N}$ and $\rho$ is as follow: $\mathrm{N}=3 \mathrm{~m}, \rho=3 \mathrm{~m} \rho_{0}$. Therefore, Figure 12 also reflects the relationship of the number of nodes $\mathrm{N}$ and the transmission delay $\mathrm{E}(\mathrm{W})$. For example, when $\rho_{0}=0.005$ and $\mathrm{N}=160$, already make $\rho=\mathrm{N} \rho_{0}=0.8$. So when the number of nodes increases, it will lead to the rapid increase of transmission delay $\mathrm{E}(\mathrm{W})$.

Because the chain network is made up of m subnets which connect in series, the transmission delay of the whole line is $\mathrm{m}$ times of the subnet transmission delay. When the number of nodes $\mathrm{N}$ in the chain network reaches a significant degree, the transmission delay of the network will increase dramatically. So there is a maximum capacity of the number of nodes in the network N. Because it is necessary to monitor that the states of all the nodes in its two hop range are idle when the node has data to transmit, so in the network, the maximum number of nodes is five, the nodes form a transmission subnet, and then form a transmission chain. Therefore, the number of tokens in the network should not exceed $\mathrm{N} / 5$, otherwise there will be interference.

\section{Conclusion}

In this paper, the network node and the corresponding transport protocol of the chain network which is suitable for power monitoring data transmission are proposed. The network-forming is convenient and costs little. The protocol is simple and easy to implement. Because the power of wireless node is limited, in the whole line, there can be multiple subnets to transmit information at the same time, and subnet will not interfere with each other, thus greatly improves the transmission efficiency, decreases the delay and reduces the bit error rate. At the same time, the token carries control information to each node, so that the nodes will not interfere with each other and realize the orderly use of the channel.

\section{About the author}

Wu Chunmei (1992), female, Master graduate student, the main research direction is communication theory and its key technology.

\section{References}

1. Li Xin. Research on Online Monitoring System of The Catenary Security Status Based on Catenary Tension and Wire Temperature[J]. Beijing Jiaotong University. 2015.

2. Li Lifen, Zhu Yongli, Yu Yonghua. Transmission of power line on-line monitoring data based on longchain tree-like wireless sensor network $[\mathrm{J}]$. Power System Technology, 2011, 35(6):199-203(in
Chinese).

3. Yin Hua, Wang Fa. Application of Wireless Mesh Technology in Power Transmission Line

Monitoring $[\mathrm{J}]$. Telecommunications for Electric Power System, 2010, 39(12) : 3609-3614.

4. LIU Yaoxian, SUN Yi, WU Xin, WEI Lei, DUAN Quansheng. A Reliable WSN Routing Method for Distribution Network Fault Detection[J]. Power System Technology, 2015, 39(12): 3609-3614(in Chinese).

5. Zhao Ziyan, Liu Jianming . A new service risk balancing based method to evaluate reliability of electric power communication network $[\mathrm{J}]$. Power System Technology, 2011, 35(10): 209-213(in Chinese).

6. Zhou Jing, Lü Tianguang, Chen Xi, et al. Study on bandwidth analysis and capacity planning of provincial dispatching digital network $[\mathrm{J}]$. Power System Technology, 2012, 36(5): 173-177(in Chinese).

7. Zhang Huafangzi . Research on Monitoring Communication System for High Voltage Transmission Lines Based on Mesh[D]. North China Electric Power University. 2013.

8. Xia Heng. Research of Wireless Streetlight Control System[D]. Harbin Institute of Technology. 2011.

9. Yang Bin, Ren Ping, Liu Yi. A Self-adaptive Token Passing Algorithm for the WTRP Network[J]. Ship Electronic Engineering. 2015, 5:47-50.

10. Li can. The Research and Application of Token Ring Protocol In The Network Communication With HighFrequency[D] . China Ship Research and Development Academy. 2014.

11. Zhang Zhidan, Huang Xiaoqing, Cao Yijia . Comprehensive data flow analysis and communication network simulation for virtual local area network-based substation[J] . Power System Technology, 2011, 35(5) :204-209(in Chinese).

12. Taner Kurtulus, Improvement and development of High-Frequency wireless token-ring protocol, Middle East Technical University, Dec 2010. 